Theoret. ehim. Acta (Berl.) 8, 427-430 (1967)

\title{
The Integral Hellmann-Feynman Theorem Applied to Hydrogen Peroxide
}

\author{
Stuart M. Rothstein, and S. M. Blinder \\ Department of Chemistry, University of Michigan, Ann Arbor, Michigan 48104
}

Received July 19, 1967

The integral Hellmann-Feynman (IHF) theorem developed by PARR [1-7] has raised hopes that the origin of barriers to internal rotation could be isolated by $a b$-initio calculations. The method is practicable for any molecule for which a wavefunction of reasonable quality is available. In such instances, the rotational barriers calculated by the IHF theorem ought to be comparable to or better than those obtained by the standard method of subtracting total SCF energies of the different conformations. To date, the successful application based on PITzER and LIPSсомв's [8] ethane wavefunctions has provided an encouraging confirmation of the usefulness of the IHF approach $[5,6]$.

Very recently three sets of molecular orbital wavefunctions for the $\mathrm{H}_{2} \mathrm{O}_{2}$ molecule have become available: the LCAO-MO-SCF-STO wave functions of KaLDOR and SHaviTT [9], the LC(Double-Zeta)AO-MO-SCE functions with scaled hydrogens of FinK and ALLEN $[10,11]$ and the LCAO-MO-SCF functions of PALKE and Prtzer [12] using exponents optimized for $\mathrm{H}_{2} \mathrm{O}$. Working independently, various workers have applied the IHF theorem to these wave functions: the first set has been treated by MELrose and PARR [13], the second by FINK and ALLEN [14], and the third by the present authors. In each case the results for the cis-trans barrier are somewhat discouraging.

Within the framework of self-consistent-field theory and the Born-Oppenheimer approximation, the IHF theorem gives the cis-trans barrier to internal rotation $\left(\Delta W_{\mathrm{IHF}}\right)$ as the sum of a nuclear-nuclear $\left(\Delta V_{n n}\right)$ and an electronic $\left(\Delta E_{e l}\right)$ barrier. In particular

$$
\Delta W_{\mathrm{IHF}}=\Delta V_{n n}+2 \sum_{i j} \frac{\left\langle\phi_{i}^{\mathrm{efs}}\left|V_{n e}^{\mathrm{cts}}-V_{n \mathrm{t}}^{\mathrm{trans}}\right| \phi_{j}^{\mathrm{trans}}\right\rangle}{\left\langle\phi_{i}^{\mathrm{ets}} \mid \phi_{j}^{\mathrm{trans}}\right\rangle}
$$

where $\phi_{i}^{x}$ is the $i^{\text {th }}$ MO for the $x$ conformation. The double summation is diagonalized by a transformation of the wave functions to corresponding orbitals (CO), $\hat{\phi}_{i}^{x}$. The addition theorem for spherical harmonics is then applied to the difference of the nuclear-electronic operators, and the transition density is expanded in a Fourier series in $\varphi[6]$. The matrices $U$ and $V$ which transform the PALKE and Pitzer MO's and CO overlaps are listed in Tabs. 1-3. These matrix elements are defined by

$$
\left|\hat{\phi}_{i}^{\text {cis }}\right\rangle=\sum_{j}\left|\phi_{j}^{\text {cis }}\right\rangle\left\langle\phi_{j}^{\text {cis }} \mid \hat{\phi}_{i}^{\text {cis }}\right\rangle \equiv \sum_{j}\left|\phi_{j}^{\text {cis }}\right\rangle U_{j i}
$$




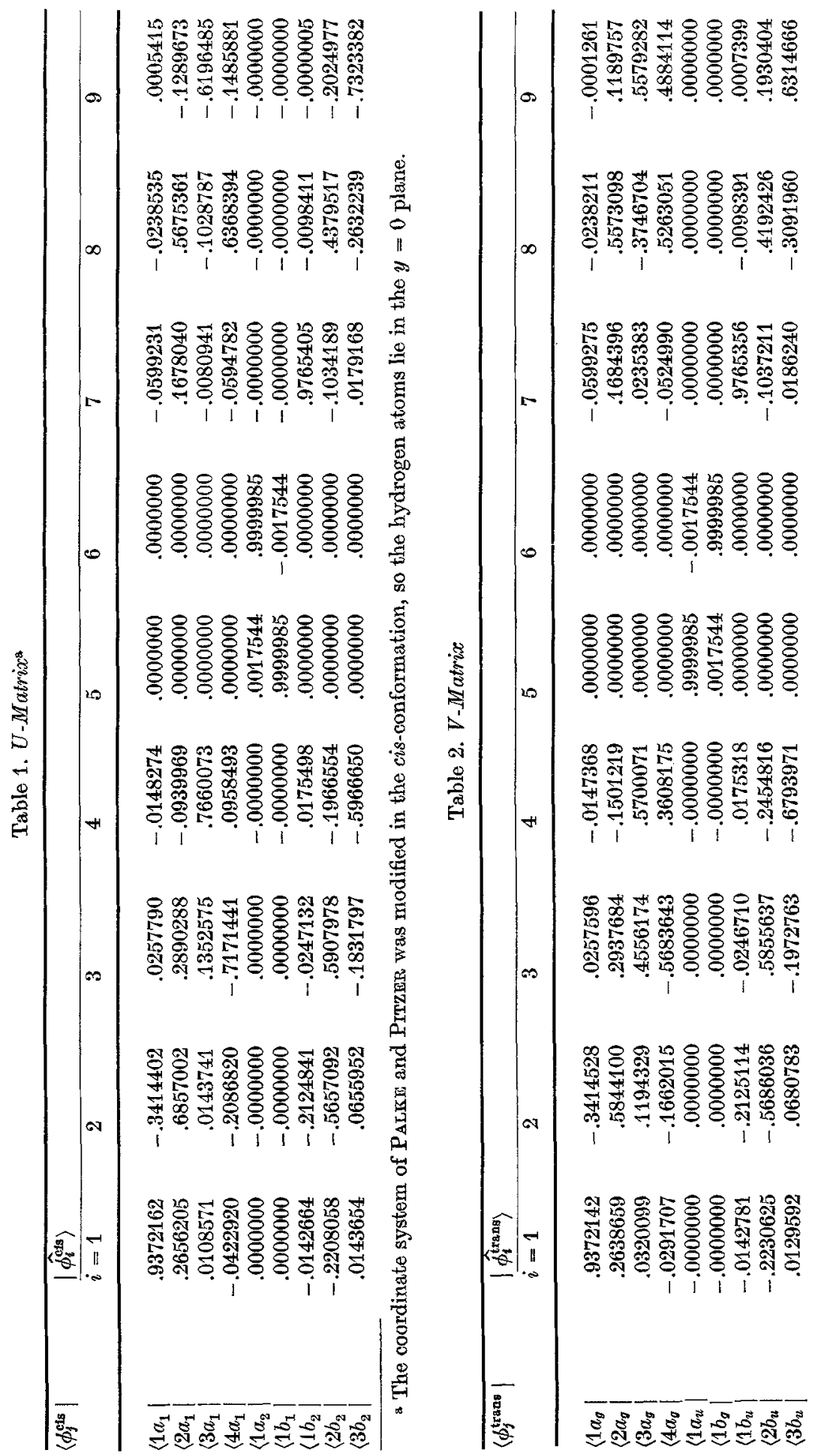




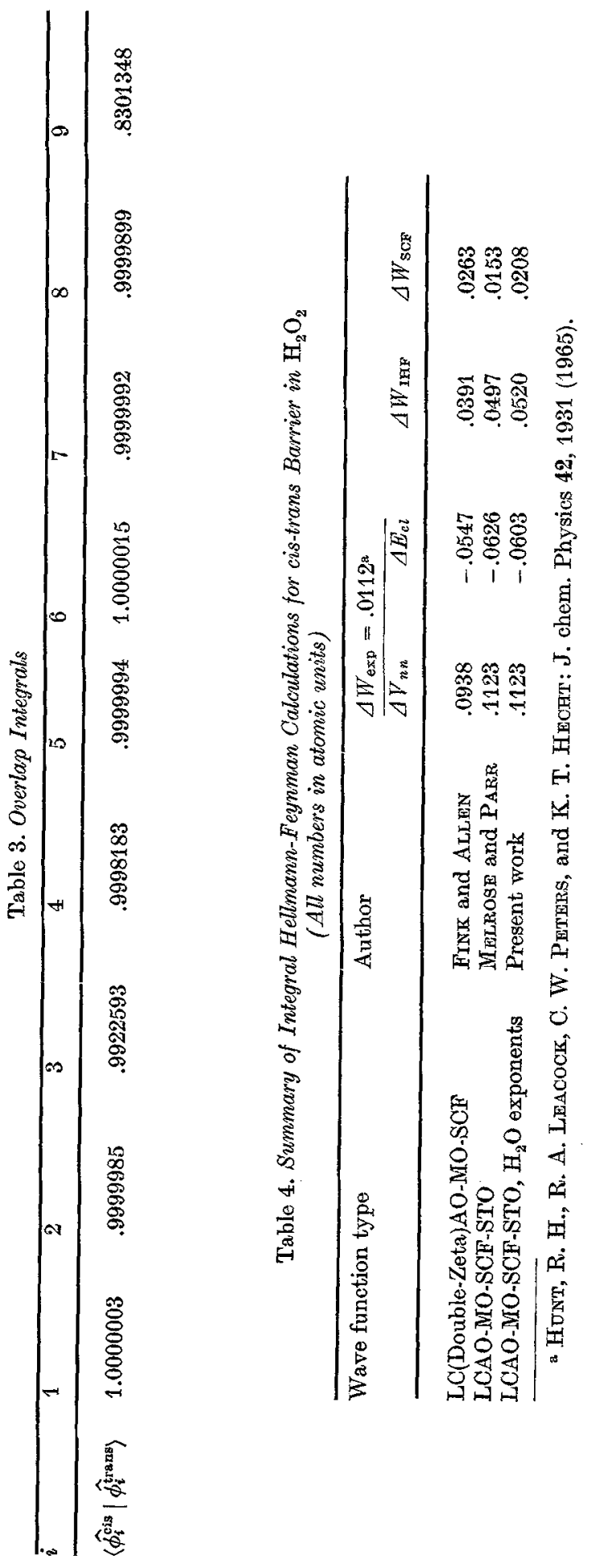


and

$$
\left|\hat{\phi}_{i}^{\text {trans }}\right\rangle=\sum_{j}\left|\phi_{j}^{\text {trans }}\right\rangle\left\langle\phi_{j}^{\text {trans }} \mid \hat{\phi}_{i}^{\text {trans }}\right\rangle \equiv \sum_{j}\left|\phi_{j}^{\text {trans }}\right\rangle V_{j i} .
$$

After Eq. (1) is diagonalized and the necessary integrations performed numerically ${ }^{\star}$, the electronic barrier is found to be -.0603 au and the total barrier to internal rotation, $.0520 \mathrm{au}$. These results and those of other workers are summarized in Tab. 4, as are the barriers obtained by direct subtraction of total SCF energies $\left(\Delta W_{\mathrm{SCF}}\right)$.

The rotation barrier as calculated from the wave functions of PALKE and PITzER is comparable to the results of FiNk and ALLEN [14] and of MELrose and ParR [13]. In each case the barrier is inferior to that obtained by the SCF subtraction method. Consequently the IHF theorem when applied to such wave functions can give no information on the barrier in $\mathrm{H}_{2} \mathrm{O}_{2}$. Recent calculations of FINK and ALLEN [14] lead to similar conclusions for a number of molecules.

Evidently, the IHF theorem must be modified in some way if it is to be applicable to wavefunctions of presently available quality.

Acknowledgements. We wish to thank Professor R. G. PARR for his cooperation and advice. The authors quoted in advance of publication are thanked for permission to use their results. SMR is particularly grateful to Professor K. T. HECHT for many illuminating discussions. Computer time for this research was supplied by the computing center of The University of Michigan.

* The integrations over $r$ and $\varphi$ are performed numerically by a $10 \times 10$ Gaussian quadrature grid and the $\varphi$ integration by a 10 point grid over each interval $(-\pi / 2 m) \leq \varphi \leq(\pi / 2 m)$ and $(n \pi / 2 m) \leq \varphi \leq[(n+2) \pi / 2 m], n$ odd, where $m(=1,3 \ldots)$ is the $m$-th order component of the transition density.

\section{References}

1. Parr, R. G.: J. chem. Physics 40, 3726 (1964).

2. KIM, H. J., and R. G. PARR: J. chem. Physics 41, 2892 (1964).

3. Karplus, M., and R. G. ParR: J. chem. Physics 38, 1547 (1963).

4. WYATT, R. E., and R. G. PARR: J. chem. Physies 41, 3262 (1964).

5. - - J. chem. Physics 43, S 217 (1965).

6. - - J. chem. Physics 44, 1529 (1966).

7. Hayes, E. F., and R. G. ParR: J. chem. Physics 44, 4650 (1966).

8. Pitzer, R. M., and W. N. Lipscomi : J. chem. Physics 39, 1995 (1963).

9. Kaldor, U., and I. SHavtTt: J. chem. Physics 44, 1823 (1966).

10. Fink, W. H., and L. C. Allen: J. chem. Physics 46, 2261 (1967).

11. - - J. chem. Physics 46, 2276 (1967).

12. Palke, W. E., and R. M. Pitzer: J. chem. Physics 46, 3948 (1967).

13. Melrose, M. P., and R. G. ParR: Theoret. chim. Acta 8, 150 (1967).

14. Fink, W. H., and L. C. Alues: J. chem. Physies 46, 3270 (1967).

Prof. Dr. S. M. BLINDER

University of Michigan

Department of Chemistry

Ann Arbor, Miehigan 48104, USA 